九州大学学術情報リポジトリ

Kyushu University Institutional Repository

\title{
Exponential Functions with Base e in Growth Analysis and Deriving Them from Rotations of Axes of Time Described using Euler's Formula
}

Shimojo, Masataka

Laboratory of Animal Feed Science, Division of Animal Science, Department of Animal and Marine

Bioresoruce Sciences, Faculty of Agriculture, Kyushu University

Ikeda, Kentaro

Research Fellow, Faculty of Agriculture, Kyushu University

Asano, Yoki

Research Fellow, Faculty of Agriculture, Kyushu University

Ishiwaka, Reiko

他

https://doi.org/10.5109/4527

出版情報：九州大学大学院農学研究院紀要. 48 (1/2)，pp.65-69，2003-10-01. Faculty of Agriculture, Kyushu University

バージョン :

権利関係 : 


\title{
Exponential Functions with Base $e$ in Growth Analysis and Deriving Them from Rotations of Axes of Time Described using Euler's Formula
}

\author{
Masataka SHIMOJO', Kentaro IKEDA ${ }^{1)}$, Yoki ASANO ${ }^{1)}$, Reiko ISHIWAKA ${ }^{1)}$, \\ Tao SHAO ${ }^{2)}$, Hiroyuki SATO ${ }^{3)}$, Manabu TOBISA, Yutaka NAKANO"), \\ Noriko OHBA $^{(5)}$, Yasukatsu YANO $^{6)}$ and Yasuhisa MASUDA \\ Laboratory of Animal Feed Science, Division of Animal Science, Department \\ of Animal and Marine Bioresource Sciences, Faculty of Agriculture, \\ Kyushu University, Fukuoka 812-8581, Japan \\ (Received June 30, 2003 and accepted July 15, 2003)
}

\begin{abstract}
This study suggests that Euler's formula including time as a variable $[\exp (i t)=\cos (t)+$ $i \sin (t)$ ] gives, through a series of $\pi / 2$ rotations of axes of time [imaginary time $(i t)$ and real time $(t)$ ], exponential functions with base $e[\exp (t)$ and $\exp (-t)]$ used for the growth analysis of ruminants and forages.
\end{abstract}

\section{INTRODUCTION}

Exponential functions with base $e$ are the function of importance to the growth analysis of ruminants (Brody, 1945; Parks, 1982; Shimojo et al., 2002b, for example) and forages (Watson, 1952; Radford, 1967; Milthorpe and Moorby, 1979; Hunt, 1990; Shimojo et al., 2002b, for example), and to the prediction of growth curves in ruminants and forages (France and Thornley, 1984, for example). It is known that Euler's formula relates, using imaginary unit, trigonometric functions to exponential functions with base $e$. Euler's formula will be changed into exponential functions with base $e$ that can be used for growth analysis, provided that imaginary unit is vanished by mathematical treatments.

The present study was designed to derive exponential functions with base $e$ from rotations of axes of time described using Euler's formula.

\section{DERIVING EXPONENTTIAL FUNCTIONS WITH BASE $e$ FROM EULER'S FORMULA}

\section{Exponential functions with base $e$ used for growth analysis}

\footnotetext{
1) Research Fellow, Faculty of Agriculture, Kyushu University

2) Visiting Research Scientist from Lanzhou Institute of Animal Science and Veterinary Medicine of CAAS, Lanzhou Gansu Province of the People's Republic of China

3) Laboratory of Animal Feed Science, Division of Animal Science, Department of Animal and Marine Bioresource Sciences, Graduate School of Bioresource and Bioenvironmental Sciences, Kyushu University

4) Kyushu University Farm, Fukuoka 811-2307

5) Research Student, School of Agriculture, Kyushu University

6) Former Technical Specialist

+ Corresponding author (E-mail: mshimojo@agr.kyushu-u.ac.jp)
} 
Exponential functions with base $e$ used for growth analysis generally take the form of $\exp (a \cdot t)$ and that of $\exp (-a \cdot t)$, where $a=$ constant $>0$ and $t=$ time (Milthorpe and Moorby, 1979). In the present study, $\exp (t)$ and $\exp (-t)$ will be taken up from the viewpoint of simplification.

\section{Euler's formula including time}

Euler's formula that includes time $(t)$ is given by

$$
\exp (i t)=\cos (t)+i \sin (t),
$$

where $\boldsymbol{i}$ =imaginary unit, $\boldsymbol{i} t=$ imaginary time, $t=$ real time.

Euler's formula includes not only real time but also imaginary time. The imaginary time is considered a tool of convenience to calculations. If this is accepted, then there will be two axes of time intersecting orthogonally each other, the axis of real time $(t)$ and the axis of imaginary time $(i t)$. Since imaginary time (it) is given by $\pi / 2$ rotation $[\times i \boldsymbol{i}$ ] of real time $(t)$, this might be expected to be followed by a series of $\pi / 2$ rotations of axes of time.

\section{$\pi / 2$ rotation of two axes of time in Euler's formula}

The $\pi / 2$ rotation of two axes of time in (1) gives

$$
\exp \{\boldsymbol{i} \cdot(\boldsymbol{i} t)\}=\cos (\boldsymbol{i} \cdot t)+\boldsymbol{i} \sin (\boldsymbol{i} \cdot t) .
$$

Then, the left-hand side of (2) is

$$
\exp \{\boldsymbol{i} \cdot(\boldsymbol{i} t)\}=\exp (-t)
$$

Using hyperbolic cosine and hyperbolic sine the right-hand side of (2) is transformed as follows:

$$
\begin{aligned}
\cos (\boldsymbol{i} \cdot t)+\boldsymbol{i} \sin (\boldsymbol{i} \cdot t) & =\cosh (t)+\boldsymbol{i} \cdot \boldsymbol{i} \sinh (t) \\
& =\cosh (t)-\sinh (t) \\
& =\frac{\exp (t)+\exp (-t)}{2}-\frac{\exp (t)-\exp (-t)}{2} \\
& =\exp (-t) .
\end{aligned}
$$

This shows that there is a reduction to the axis of real time $(-t)$ by $\pi / 2$ rotation of both imaginary time (it) and real time $(t)$ axes in Euler's formula.

\section{$\pi / 2$ rotation of the axis of time in $\exp (-t)$}

The $\pi / 2$ rotation of the axis of real time in $\exp (-t)$ gives

$$
\exp \{\boldsymbol{i} \cdot(-t)\}=\cos (-t)+\boldsymbol{i} \sin (-t) .
$$

It is shown that $\pi / 2$ rotation of the axis of real time $(-t)$ in $\exp (-t)$ gives, through an appearance of the conjugate complex to Euler's formula, the axis of imaginary time (-it) and that of real time $(-t)$.

\section{$\pi / 2$ rotation of two axes of time in the conjugate complex to Euler's formula}

The $\pi / 2$ rotation of two axes of time in (5) gives 


$$
\exp [\boldsymbol{i} \cdot\{\boldsymbol{i} \cdot(-t)\}]=\cos \{\boldsymbol{i} \cdot(-t)\}+\boldsymbol{i} \sin \{\boldsymbol{i} \cdot(-t)\} .
$$

Then, the left-hand side of (6) is

$$
\exp [\boldsymbol{i} \cdot\{\boldsymbol{i} \cdot(-t)\}]=\exp (t) .
$$

Transforming the right-hand side of (6) using hyperbolic cosine and hyperbolic sine gives

$$
\begin{aligned}
\cos \{\boldsymbol{i} \cdot(-t)\}+\boldsymbol{i} \sin \{\boldsymbol{i} \cdot(-t)\} & =\cosh (-t)+\boldsymbol{i} \cdot \boldsymbol{i} \sinh (-t) \\
& =\cosh (t)+\sinh (t) \\
& =\frac{\exp (t)+\exp (-t)}{2}+\frac{\exp (t)-\exp (-t)}{2} \\
& =\exp (t) .
\end{aligned}
$$

This shows that there is a reduction to the axis of real time $(t)$ by $\pi / 2$ rotation of both imaginary time (-it) and real time $(-t)$ axes in the conjugate complex to Euler's formula.

\section{$\pi / 2$ rotation of the axis of time in $\exp (t)$}

The $\pi / 2$ rotation of the axis of real time in $\exp (t)$ gives a return to the start, namely Euler's formula,

$$
\exp (i t)=\cos (t)+i \sin (t)
$$

This anti-clockwise cycle, using $\pi / 2$ rotation [Xi], of Euler's formula, (1) $\rightarrow[(3)$ and $(4)] \rightarrow(5) \rightarrow[(7)$ and (8)] $\rightarrow(1)$, is shown in Fig. 1. There is also a clockwise cycle, using $-\pi / 2$ rotation $[\times(-i)]$, of the conjugate complex to Euler's formula in order to obtain $\exp (-t)$ and $\exp (t)$.

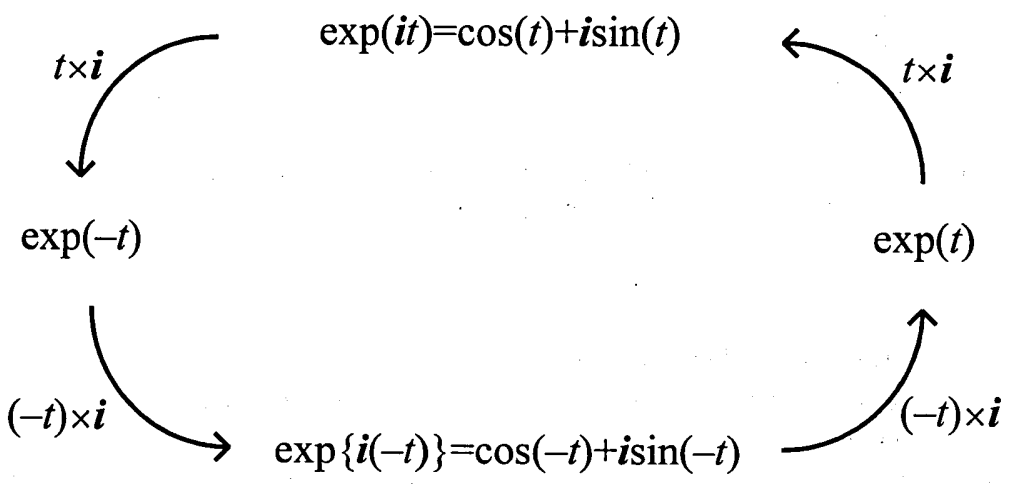

Fig. 1. An anti-clockwise cycle in Euler's formula using $\pi / 2$ rotation $[\times i]$ of axes of time.

\section{Comparison between $\exp (t)$ and $\exp (-t)$}

When the passage of time $(t)$ is shown by $0 \rightarrow 1 \rightarrow 2 \rightarrow 3$, for example, there is a moving forward in time $(0 \rightarrow 1 \rightarrow 2 \rightarrow 3)$ for $\exp (t)$, but $\exp (-t)$ shows a moving backward in time $(0 \rightarrow-1 \rightarrow-2 \rightarrow-3)$ that comes from an interpretation as $\exp \{1(-t)\}$. 
In addition, if $\exp (t)$ is regarded as $\exp \{-1(-t)\}$, then this will show a moving backward in time. The existence of Euler's formula and its conjugate complex between $\exp (t)$ and $\exp (-t)$, which is shown in $(1) \sim(8)$, suggests that imaginary time and real time axes intersecting orthogonally each other separate the moving forward in time from the moving backward in time.

As shown in (1) $\sim(4)$, the anti-clockwise $\pi / 2$ rotation $[\times i]$ of two axes of time in Euler's formula gives $\exp (-t)$, a moving backward in time. Then the clockwise $\pi / 2$ rotation $[\times(-\boldsymbol{i})]$ of two axes of time in Euler's formula gives $\exp (t)$, a moving forward in time as shown in the following calculation and in Fig. 2. Thus,

$$
\begin{aligned}
\exp \{-\boldsymbol{i} \cdot(\boldsymbol{i} t)\} & =\cos (-\boldsymbol{i} \cdot t)+\boldsymbol{i} \sin (-\boldsymbol{i} \cdot t) \\
& =\cos \{(\boldsymbol{i} \cdot(-t)\}+\boldsymbol{i} \sin \{(\boldsymbol{i} \cdot(-t)\},
\end{aligned}
$$

therefore, the left-hand side of (9) is

$$
\exp \{-\boldsymbol{i} \cdot(\boldsymbol{i} t)\}=\exp (t)
$$

and the right-hand side of (9) is

$$
\begin{aligned}
\cos \{\boldsymbol{i} \cdot(-t)\}+\boldsymbol{i} \sin \{\boldsymbol{i} \cdot(-t)\} & =\cosh (-t)+\boldsymbol{i} \cdot \boldsymbol{i} \sinh (-t) \\
& =\cosh (t)+\sinh (t) \\
& =\frac{\exp (t)+\exp (-t)}{2}+\frac{\exp (t)-\exp (-t)}{2} \\
& =\exp (t) . \\
&
\end{aligned}
$$

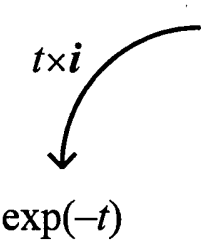

Fig. 2. A clockwise $\pi / 2$ rotation $[\times(-\boldsymbol{i})]$ and an anti-clockwise $\pi / 2$ rotation $[\times \boldsymbol{i}]$ of two axes of time in Euler's formula.

There is also another way: the clockwise $\pi / 2$ rotation $[\times(-i)]$ and anti-clockwise $\pi / 2$ rotation $[\times \boldsymbol{i}$ ] of two axes of time in the conjugate complex to Euler's formula lead to $\exp (-t)$ and $\exp (t)$, respectively.

However, in the actual growth analysis of ruminants and forages, $\exp (t)$ and $\exp (-t)$ are regarded as $\exp (1 \cdot t)$ and $\exp (-1 \cdot t)$, respectively, in order to show the moving forward in time (Milthorpe and Moorby, 1979).

\section{EULER'S FORMULA AND MACRO-ASPECTS OF RUMINANT AGRICULTURE}

The present study suggests that exponential functions with base $e$ used for the 
growth analysis of ruminants and forages are derived from a series of $\pi / 2$ rotations of axes of time described using Euler's formula. The growth of forages and ruminants is a macro-aspect of great importance to ruminant agriculture, as well as field-forage-ruminant relationships (Shimojo et al., 2002a, 2003a, b).

It is known that Euler's formula shows a spiral when described stereographically (Yoshida, 2000), which might be associated with micro-structures in ruminants and forages. This will be taken up in the following paper in this issue.

\section{REFERENCES}

Brody, S. 1945 Time relations of growth of individuals and populations. In "Bioenergetics and growth", Reinhold Publishing Corporation, New York, pp. 484-574

France, J. and J. H. M. Thornley 1984 Growth functions. In "Mathematical Models in Agriculture - A Quantitative Approach to Problems in Agriculture and Related Sciences", Butterworth \& Co (Publishers) Ltd, London, pp. 75-94

Hunt, R. 1990 Basic Growth Analysis. Unwin Hyman Ltd., London.

Milthorpe, F. L. and J. Moorby 1979 Some commonly used functions. In "An introduction to crop physiology", Cambridge University Press, Cambridge, pp. 224-227

Parks, J. R. 1982 A theory of feeding and growth of animals. Springer-Verlag, New York.

Radford, P. J. 1967 Growth analysis formulae-their use and abuse. Crop Sci., 7: 171-175

Shimojo, M., K. Ikeda, Y. Asano, R. Ishiwaka, M. Tobisa, T. Shao, N. Ohba, H. Sato, Y. Matsufuji, Y. Yano and Y. Masuda 2002a An intersection of ruminant production and forage production. J. Fac. Agr., Kyushu Univ., 46: 321-329

Shimojo, M., Y. Asano, K. Ikeda, R. Ishiwaka, T. Shao, N. Ohba, H. Sato, Y. Matsufuji, M. Tobisa, Y. Yano and Y. Masuda 2002b Basic growth analysis and symmetric properties of exponential function with base e. J. Fac. Agr., Kyushu Univ., 47: 55-60

Shimojo, M., K. Ikeda, Y. Asano, R. Ishiwaka, T. Shao, H. Sato, M. Tobisa, Y. Nakano, N. Ohba, Y. Yano and Y. Masuda 2003a A symbolic representation of field-forage-ruminant relationships using polar form on the complex plane. J. Fac. Agr., Kyushu Univ., 47: 359-366

Shimojo, M., Y. Asano, K. Ikeda, R. Ishiwaka, T. Shao, H. Sato, M. Tobisa, Y. Nakano, N. Ohba, Y. Yano and Y. Masuda 2003b Complex representation of field-forage-ruminant relationships using symmetric properties of Euler's formula. J. Fac. Agr., Kyushu Univ., 47: 367-372

Watson, D. J. 1952 The physiological basis of variation in yield. Adv. Agron., 4: 101-145

Yoshida, T. 2000 Emotion for imaginary number. Tokai University Press, Tokyo. (Written in Japanese) 